\title{
Cost of inpatient rehabilitation care in the Department of Veterans Affairs
}

\author{
Todd H. Wagner, PhD; ${ }^{1-3^{*}}$ Samuel S. Richardson, BA; ${ }^{1}$ Bruce Vogel, PhD; ${ }^{4}$ Kristen Wing, BA; ${ }^{4}$ \\ Mark W. Smith, $\mathbf{P h D}^{\mathbf{1 - 2}}$ \\ ${ }^{1}$ Department of Veterans Affairs (VA) Health Economics Resource Center, Menlo Park, CA; ${ }^{2}$ Center for Primary Care \\ and Outcomes Research, Stanford University, Stanford, CA; ${ }^{3}$ Department of Health Research and Policy, Stanford Uni- \\ versity, Stanford, CA; ${ }^{4}$ Rehabilitation Outcomes Research Center, VA Medical Center, Gainesville, FL
}

\begin{abstract}
We investigated the determinants of inpatient rehabilitation costs in the Department of Veterans Affairs (VA) and examined the relationship between length of stay (LOS) and discharge costs using data from VA and community rehabilitation hospitals. We estimated regression models to identify patient characteristics associated with specialized inpatient rehabilitation costs. VA data included 3,535 patients discharged from 63 facilities in fiscal year 2001. We compared VA costs to community rehabilitation hospitals using a sample from the Uniform Data System for Medical Rehabilitation of 190,112 patients discharged in 1999 from 697 facilities. LOS was a strong predictor of cost for VA and non-VA hospitals. Functional status, measured by Functional Independence Measure (FIM) scores at admission, was statistically significant but added little explanatory value after controlling for LOS. Although FIM scores were associated with LOS, FIM scores accounted for little variance in cost after controlling for LOS. These results are most applicable to researchers conducting cost-effectiveness analyses.
\end{abstract}

Key words: average costs, billing, charges, cost, economics, micro-cost methods, reimbursement, rehabilitation, VA, veterans.

\section{INTRODUCTION}

The Department of Veterans Affairs (VA) provided nearly 13,000 veterans with 445,000 days of specialized rehabilitation care in fiscal year (FY) 2004, at a cost of $\$ 453$ million [1]. This represented a 4 percent increase in days and a 6 percent increase in costs from FY2002 [1]. At a time when VA inpatient services have been declining [2-4], the slight growth in specialized rehabilitation services reflects a rapidly aging veteran population [5], whereby older veterans are more likely than younger veterans to suffer from injurious falls and stroke, two conditions needing specialized rehabilitation. These increases also reflect injuries sustained by military personnel deployed in Iraq and Afghanistan who are receiving VA care.

Despite the magnitude of VA rehabilitation services and their importance to veterans, we know little about the determinants of costs and how the costs of VA rehabilitation services compare with the costs of rehabilitation services in non-VA community facilities. Until recently, such analyses would not have been possible because of a

Abbreviations: $\mathrm{CPI}=$ consumer price index, DSS $=$ Decision Support System, FIM = Functional Independence Measure, FY = fiscal year, GLM = generalized linear models, HERC $=$ Health Economics Resource Center, LOS = length of stay, SCI = spinal cord injury, $\mathrm{SD}=$ standard deviation, UDSMR = Uniform Data System for Medical Rehabilitation, VA = Department of Veterans Affairs.

*Address all correspondence to Todd $\mathrm{H}$. Wagner, $\mathrm{PhD}$; Department of Veterans Affairs Palo Alto, 795 Willow Rd, 152-MPD, Menlo Park, CA 94025; 650-493-5000, ext 22048; fax: 650-617-2639. Email: twagner@stanford.edu DOI: 10.1682/JRRD.2005.10.0162 
lack of patient-level VA cost data. The creation of two such databases, the Health Economics Resource Center (HERC) average cost data sets and the Decision Support System (DSS) National Data Extracts, now enable researchers to study encounter-level rehabilitation costs in VA facilities. These two cost databases use different methods to estimate the cost of rehabilitation care. In the HERC data, the cost of a stay is calculated by multiplying each patient's length of stay (LOS) by a national per diem cost estimate. The HERC cost does not depend on patient or clinical characteristics such as age, sex, diagnosis, comorbidities, initial functional status, or functional gain during rehabilitation. DSS costs for inpatient stays are estimated using direct measurement, also known as activity-based costing. DSS cost estimates should reflect patient and clinical characteristics to the extent that these factors affect the type and quantity of services provided.

DSS data offer considerable opportunities for researchers. The data on rehabilitation patients, however, have yet to be compared with other VA or non-VA sources. Preliminary work on patients with acute myocardial infarction suggests that validation of DSS cost estimates is essential prior to use of the DSS data for research purposes [6]. Furthermore, Wagner and Velez identified differences between DSS and HERC inpatient rehabilitation cost estimates at an aggregate level [7]. The lack of accurate and valid VA inpatient rehabilitation cost data limits rehabilitation researchers' ability to conduct costeffectiveness analyses of rehabilitation care.

The first objective of this study was to compare the HERC and DSS data so that researchers would be aware of the advantages and limitations of using these data sets in research. In particular, we investigated the relationship between LOS and costs because LOS is the sole determinant of HERC costs. In 1979, Luke found the correlation coefficient between LOS and total charges ranged from 0.81 to 0.95 [8], but important characteristics of inpatient care may have changed in the past 25 years, and Luke's data did not come from VA patients. Other studies have used LOS as a surrogate for costs [9-18], but the ability to predict costs accurately with LOS is relatively unknown.

The second objective was to investigate predictors of VA and non-VA rehabilitation costs. By comparing the predictors, including the role of LOS, we can assess the construct validity of VA costs.

\section{METHODS}

\section{Conceptual Model}

Inpatient rehabilitation facilities purchase inputs include labor, equipment, and supplies. These inputs are used to treat patients - hence, patient care is the output. The relationship between inputs and outputs can be characterized by a production function. Outputs can be assessed by measuring quantity of goods or their costs. Cost analysis is often done when there is heterogeneity in the quantity of service. In this article, we analyze costs.

Different types of costs exist. Labor and supplies vary in their use across patients and within patients over time and so are labeled variable costs. Capital costs such as buildings and major equipment are fixed in the short term, and hence they are called fixed costs. Together, the variable and fixed costs make up the total cost.

Hospitals use these fixed and variable inputs to treat patients. The relationship between inputs and costs can be examined with an economic production function. Most empirical studies of hospital production functions use hospital discharge data sets. These data sets provide a wealth of diagnostic clinical information but do not fully enumerate all the inputs required to treat a patient. For example, most inpatient discharge data sets do not identify the time a provider spent with a patient. To address this problem, researchers use proxies for resource use.

The most common proxy is LOS. Including LOS in a production function provides information on the fixed costs (e.g., the hospital's capital cost). LOS can also provide information on variable costs that recur during a hospitalization. Examples of recurring costs include therapies that occur many times during the stay, such as physical therapy, or room and board charges.

Researchers often include clinical and patient information in economic production functions as well. These variables can also act as proxies for resource use. To date, costs have been associated with diagnosis [9,12-14,1820], functional status at admission [9,11-16,18-23], age $[9,11,13,16,18,20,22,24]$, length of a preceding acute hospitalization [9,13-14,22], comorbidities [20-21], presence of medical tubes [15,21], and marital status [16]. These are proxies for resource use because a patient's clinical and sociodemographic status directly affects quantity of services provided, which in turn affects the cost.

When clinical and patient factors are included in a production function, the results need to be interpreted 
with caution. Little or no association may exist between patient severity and costs when other variables, such as LOS, are in the models. This is especially true when sicker patients have longer stays than healthier patients.

Production functions should not be confused with conceptual models underlying healthcare financing studies. Carter et al., for example, set out to create a prospective payment system for inpatient rehabilitation [19]. They used admission information to create a system that rewarded efficiency (i.e., to provide the same care using fewer resources or to make more care using the same resources). Because LOS is not known at admission, it is not part of these models.

\section{Sample}

We used three data sets in our analyses: HERC and DSS data from FY 2001 and Uniform Data System for Medical Rehabilitation (UDSMR) community rehabilitation data from 1999. DSS data from FY01 and later are believed to be more accurate than data from earlier years. Although 2001 data from UDSMR would have been preferable, the most recent data available were from 1999. We adjusted UDSMR dollar values to 2001 using the Bureau of Labor Statistics general consumer price index (CPI) for urban areas before the comparison. The study protocol was approved by the Stanford University Institutional Review Board.

\section{Department of Veterans Affairs Data}

DSS was designed to provide accurate cost estimates of all VA services in a given FY. DSS employs an activity-based costing system that extracts expenditure data from the VA general ledger and the VA payroll system and allocates costs to patient care departments and to patient encounters based on staff activities. DSS identifies the quantity and local price of each input used in the production of inpatient rehabilitation care. The costs of all the inputs are then summed to find the total cost of an encounter. The system reflects variation in local prices and in the supply of technology. This accounting method is generally considered the gold standard for cost determination [25].

The HERC costs for rehabilitation stays are estimated from a model that calculates the national average daily cost as the quotient of total rehabilitation expenses and total rehabilitation days for the nation. This is an average daily rate or the national average cost of care for one day. The estimated cost of a stay is then the product of the average daily rate times a patient's LOS [26]. The costs are based on all rehabilitation costs nationwide and so the estimated costs reflect national averages, not costs pertaining to a specific geographic area.

For both the DSS and HERC data, we used administrative codes to identify patients receiving specialized rehabilitation. We excluded patients located in other bedsections (a VA bed section is akin to a ward), such as medicine and psychiatry, who may have received some rehabilitation care while in these other bed sections. Therefore, our sample reflects care in specialized rehabilitation units. We used data from all patients discharged from 63 VA rehabilitation facilities throughout the country in FY01.

VA provides care in general rehabilitation, spinal cord injury (SCI), and blind rehabilitation. We merged rehabilitation discharge records with Functional Independence Measure (FIM) scores stored in the in the VA Functional Status and Outcomes Database. FIM scores were available only for general rehabilitation patients, and thus SCI and blind rehabilitation discharges were excluded from our VA sample. We merged FIM scores with 76 percent of general rehabilitation discharges for a total of 3,535 records. This number does not represent such unique patients as those who had more than one discharge in 2001.

\section{Uniform Data System for Medical Rehabilitation Data Set}

The 1999 UDSMR data set included national data for 334,008 patients discharged from 697 community rehabilitation facilities. UDSMR records report charges (not costs), patient demographic information, and characteristics of stays such as diagnosis, LOS, and FIM scores at admission and discharge. We excluded 60,250 records that included physician costs or did not confirm the inclusion of physician costs. We excluded 18,044 records that were missing charge information; another 2,848 cases were deleted because of missing data on the LOS, FIM, age, sex, and in-hospital death variables. Then, to make UDSMR data more comparable to VA data, we excluded 3,549 UDSMR records for patients under the age of 18 and patients treated in Hawaii or Alaska. The resulting UDSMR data set had 249,317 cases.

The UDSMR data report charges whereas DSS data report costs. To be consistent with VA data, we linked the UDSMR data to the 1999 Medicare Cost Reports to costadjust the charges. This link succeeded for 190,112 (76\%) of the 249,317 records. No differences existed in 
terms of LOS, admission FIM score, or sex between the 190,112 and 249,317 records. Our analytic data set included all 190,112 records.

\section{Variables}

We calculated LOS using the admission date and discharge date, subtracting any program interruption days. In our regression models, we used the natural log of LOS because of its skewed distribution. The relationship between cost and LOS can be nonlinear. To account for this, we included a variable indicating an atypically short stay (LOS $<4$ days) because higher costs may be incurred at the beginning of a stay [20]. In addition, we included variables indicating whether the patient died in the hospital, was discharged to home, or was discharged elsewhere.

The admission FIM score was measured within 3 days of rehabilitation admission; it includes subscales for motor and cognitive functional independence. Higher FIM scores indicate higher levels of functional independence. We scaled the motor and cognitive functional independence scales between 1 and 7, and then divided the values into three categories: low (1.0-2.5), medium (2.5-5.5), and high (5.5-7.0). Therefore, we divided patients into nine categories ( 3 motor groups $\times 3$ cognitive groups) based on admission FIM scores. We grouped age into categories $(17-45,46-65,66-75,76-85$, and $86+$ ) to allow for nonlinearities. To account for regional variation in prices, we merged our data with the 2001 Medicare wage index for the hospital's region.

\section{Analysis}

In all our regression models, we used ordinary least squares with the natural log of costs (VA) or log of costadjusted charges (UDSMR) as the dependent variable. Because costs and LOS were log-transformed, the beta coefficient on LOS represents the percent change in cost resulting from a 1 percent change in LOS. We used the smearing estimator with a correction for LOS-related heteroscedasticity when transforming the logged estimates back to dollars [27-28]. We used robust standard errors to control for potential heteroscedasticity. We used generalized linear models (GLMs) with a log link and gamma distribution, following Blough et al. [29], as a sensitivity analysis. We performed all analyses using Stata 8.2 (Stata Corporation LP, College Station, Texas).

\section{RESULTS}

\section{Sample Characteristics}

Table 1 shows descriptive statistics for the VA and UDSMR data. VA rehabilitation patients were younger than community patients, more likely to be male, and more likely to have an atypically short stay ( $<4$ days).

VA patients have higher overall FIM scores compared with patients in the UDSMR data (a higher FIM score means greater functioning). Although minor differences existed between VA and non-VA patients with respect to their cognitive FIM scores, the difference in motor score drove the overall difference in FIM scores. Among veterans, 14.7 percent had a FIM motor score of greater than 5.5, whereas only 1.7 percent of the UDSMR cases had a score of greater than 5.5. Similarly, 18.2 percent of UDSMR cases were categorized with a motor FIM score <2.5, whereas only 11.6 percent of veterans had a similar score.

\section{Average Cost Per Day}

The unadjusted average cost was $\$ 763$ a day, $\$ 1,329$, and $\$ 988$ for the UDSMR, HERC, and DSS data, respectively, in 2001 dollars. The HERC daily costs did not vary, by construction. The UDSMR data had a standard deviation (SD) of $\$ 281$ and a range of $\$ 85$ to $\$ 7,787$. The SD of the DSS data was $\$ 644$, with a range of $\$ 83$ to $\$ 22,633$. The maximum DSS value was an outlier; no other DSS records averaged over $\$ 7,000$ a day. As we highlight in the discussion, a maximum cost per day of $\$ 22,633$ may be a data error or it may be a valid high-cost visit. Researchers should not discard outliers assuming they are data input errors. Costs are frequently skewed and valid high-cost visits may be critical to the analysis.

\section{Discharge Costs}

Bivariate analysis of DSS data showed that the log of LOS explained 83 percent of the total variance in the log of total cost (not shown). Log-transformed LOS explained 81 percent of the total variance in the log of cost-adjusted charges with the UDSMR data. By construction, the HERC costs varied in direct proportion to LOS.

In multivariate analysis with the UDSMR and DSS data, LOS explained most of the variance in cost-adjusted charges and costs, respectively. Age, sex, atypical short stay, not discharged to home, and FIM scores were all statistically associated with cost-adjusted charges (Table 2). 
Table 1.

Department of Veterans Affairs (VA) and Uniform Data System for Medical Rehabilitation (UDSMR) descriptive statistics. Data presented as percentages unless otherwise noted.

\begin{tabular}{lcc}
\hline \multicolumn{1}{c}{ Characteristics } & VA & USDMR \\
\hline Observations $(n)$ & 3,535 & 190,112 \\
LOS & $15.5 \pm 12.2$ & $15.5 \pm 12.3$ \\
Days (Mean \pm SD) & 4.8 & 3.5 \\
Atypical Short Stay (LOS $<4)$ & & \\
Discharged Status & 88.2 & 81.9 \\
Discharged to Home & 0.3 & 0.3 \\
Died in Hospital & 11.5 & 17.8 \\
Transferred or Discharged Elsewhere & & 9.6 \\
Age & 7.9 & 22.3 \\
17-45 & 39.9 & 28.5 \\
46-65 & 25.4 & 30.5 \\
66-75 & 24.4 & 9.1 \\
76-85 & 2.4 & 58.1 \\
86+ & 4.0 & \\
Female (\%) & & $72.9 \pm 18.8$ \\
Functional Independence Measure & $82.4 \pm 21.3$ & 18.2 \\
Score At Admission (Mean \pm SD) & 11.6 & 80.1 \\
Motor $<2.5$ & 73.7 & 1.7 \\
Motor 2.5-5.5 & 14.7 & 7.0 \\
Motor $>5.5$ & 5.3 & 30.6 \\
Cognitive $<2.5$ & 27.6 & 62.4 \\
Cognitive 2.5-5.5 & 67.1 & $0.99 \pm 0.13$ \\
Cognitive $>5.5$ & $1.06 \pm 0.16$ & \\
Medicare Wage Index (Mean \pm SD) & & \\
\hline \hline
\end{tabular}

However, the adjusted $R^{2}$ in the regression model only increased to 84 from 81 percent in the bivariate analysis when other covariates, including FIM, were added.

To show the predictive power of the FIM scores, we used the regression model to predict costs at different FIM scores, holding LOS, atypical short stay, discharge status, sex, and age constant at the sample mean. We allowed for differences in costs between UDSMR and DSS. The unadjusted and adjusted costs are shown in Table 3. Of particular interest is the much lower FIMrelated variation in adjusted costs compared with unadjusted costs. This reflects the fact that LOS varies with costs, and once we controlled for LOS, the variation in costs dramatically decreased. On average, patients with higher FIM scores had lower adjusted discharge costs, but costs did not always decrease as one would expect and the reductions were relatively modest.
The GLM sensitivity analysis confirmed that the results were highly robust. LOS was the primary determinant of costs. The addition of other variables, while statistically significant, was not as important as LOS in explaining costs. Reanalyzing the DSS data without the outlier (i.e., regression constrained to cases with a per diem cost of $<\$ 7000$ ) had no effect on the results; therefore, we present the results with the outliers included.

\section{DISCUSSION}

LOS explained approximately 80 percent of the variance in cost of inpatient specialized rehabilitation stays. These results are consistent with Luke, whose results were published over 25 years ago [8]. Patient and clinical factors were statistically significant predictors of cost, but they added little explanatory value as indicated by only a 
JRRD, Volume 43, Number 7, 2006

Table 2.

Determinants of logged costs using a multivariate regression model. Robust standard error in parentheses.

\begin{tabular}{|c|c|c|}
\hline Characteristics & VA DSS Costs & UDSMR Cost-Adjusted Charges \\
\hline Natural Log of LOS & $0.942^{*}(0.010)$ & $0.986^{*}(0.001)$ \\
\hline Atypical Short Stay (LOS <4) & $0.061(0.033)$ & $0.085^{*}(0.005)$ \\
\hline Discharge Status & & \\
\hline Discharged to Home (Ref) & - & - \\
\hline Died in Hospital & $0.178^{\dagger}(0.090)$ & $0.154^{*}(0.017)$ \\
\hline Transferred or Discharged Elsewhere & $-0.038^{*}(0.015)$ & $0.055^{*}(0.002)$ \\
\hline \multicolumn{3}{|l|}{ Age } \\
\hline $17-45$ & $-0.028(0.022)$ & $0.097^{*}(0.003)$ \\
\hline 46-65 & $-0.003(0.013)$ & $0.046^{*}(0.002)$ \\
\hline 66-75 (Ref) & - & - \\
\hline $76-85$ & $-0.011(0.014)$ & $-0.032^{*}(0.002)$ \\
\hline Motor Low, Cognitive High & $0.022(0.038)$ & $-0.049^{*}(0.005)$ \\
\hline Motor Medium, Cognitive Low & $-0.019(0.046)$ & $-0.086^{*}(0.006)$ \\
\hline Motor Medium, Cognitive Medium & $-0.026(0.031)$ & $-0.092^{*}(0.004)$ \\
\hline Motor Medium, Cognitive High & $-0.087^{*}(0.031)$ & $-0.141^{*}(0.004)$ \\
\hline Motor High, Cognitive Low & $0.012(0.065)$ & $-0.170 *(0.037)$ \\
\hline Motor High, Cognitive Medium & $-0.053(0.044)$ & $-0.102^{*}(0.012)$ \\
\hline Motor High, Cognitive High & $-0.142^{*}(0.036)$ & $-0.160^{*}(0.008)$ \\
\hline Medicare Wage Index & $0.445^{*}(0.030)$ & $0.601^{*}(0.007)$ \\
\hline Constant & $6.573^{*}(0.058)$ & $6.127^{*}(0.008)$ \\
\hline
\end{tabular}

slight increase in $R^{2}$. This strong relationship between LOS and costs indicates that the majority of inpatient rehabilitation costs are fixed or recurring. Many rehabilitation services, in fact, occur daily for a set period of time. Hence, the relationship between LOS and total costs may not be surprising. LOS does not perfectly explain costs because rehabilitation services vary, in terms of treatment type, intensity, quantity, and price, because some patients use ancillary services (e.g., imaging) and because accounting practices are not entirely uniform.

FIM scores were significantly associated with discharge costs. Consistent with work by Carter and colleagues [19], we find evidence that costs vary more across the spectrum of FIM motor scores than FIM cog- nitive scores. When we controlled for LOS, FIM scores remained significant determinants of costs, but they had little explanatory power in terms of variance. This effect is not entirely surprising because FIM is highly associated with LOS. Hence, FIM affects discharge costs mostly through its association with LOS, although FIM scores were significantly associated with costs after controlling for LOS.

VA patients had fewer functional limitations and, in particular, better motor functioning, than non-VA patients. After adjusting for FIM and LOS, VA costs were higher than UDSMR cost-adjusted charges. While this is striking, caution is needed in interpreting this difference. The difference in costs could relate to differences in treatment 
Table 3.

Predicted average total costs (2001 \$US) by admission Functional Independence Measure cognitive and motor scores.

\begin{tabular}{|c|c|c|c|c|c|c|}
\hline \multirow{2}{*}{ Cost Data } & \multicolumn{2}{|c|}{ Cognitive Low } & \multicolumn{2}{|c|}{ Cognitive Medium } & \multicolumn{2}{|c|}{ Cognitive High } \\
\hline & $\$$ & \% Change* & $\$$ & \% Change* & $\$$ & \% Change* \\
\hline \multicolumn{7}{|l|}{$\overline{\text { UDSMR }}$} \\
\hline Unadjusted Costs & & & & & & \\
\hline Motor Low & 24,306 & 354 & 20,478 & 283 & 22,162 & 314 \\
\hline Motor Medium & 14,030 & 162 & 12,040 & 125 & 8,993 & 68 \\
\hline Motor High & 7,274 & 36 & 7,340 & 37 & 5,351 & 0 \\
\hline Motor Low & 10,416 & 17 & 9,957 & 12 & 9,910 & 11 \\
\hline Motor Medium & 9,529 & 7 & 9,466 & 6 & 9,001 & 1 \\
\hline Motor High & 8,756 & -2 & 9,378 & 5 & 8,890 & 0 \\
\hline \multicolumn{7}{|l|}{ DSS } \\
\hline \multicolumn{7}{|l|}{ Unadjusted Costs } \\
\hline Motor Low & 13,618 & 13 & 14,102 & 17 & 13,994 & 16 \\
\hline Motor Medium & 13,509 & 12 & 13,496 & 12 & 12,656 & 5 \\
\hline Motor High & 13,533 & 12 & 13,192 & 9 & 12,083 & 0 \\
\hline \multicolumn{7}{|c|}{$\begin{array}{l}\text { Note: Health Economics Resource Center costs vary only by length of stay and therefore were not included. } \\
{ }^{*} \text { Represents percent change from cognitive high and motor high group. } \\
{ }^{\dagger} \text { Adjusted costs were calculated from regression model that removed variation associated with: length of stay, atypical short stay, discharge status, sex, and age } \\
\text { Variables were held at their sample means. In regression, adjusted costs were allowed to vary by DSS and UDSMR. Adjusted costs were corrected using } \\
\text { heteroscedastic-smearing estimator. } \\
\text { DSS = Decision Support System, UDSMR = Uniform Data System for Medical Rehabilitation. }\end{array}$} \\
\hline
\end{tabular}

intensity, provider efficiency, or other institutional factors [30]. It could also relate, in part, to problems with comparing cost-adjusted charges and costs, or in using the CPI to inflate the 1999 UDSMR data to 2001 dollars, the year of the VA data. The general CPI reports inflation of 5.9 percent and this may have underestimated inflation. The medical services CPI reports inflation of 8.3 percent, but estimating inflation for medical care is fraught with problems as new technologies emerge and the quality of care changes over time [31]. More research is needed to understand if real differences in costs exist between VA and non-VA rehabilitation providers and what is driving these differences.

Rehabilitation researchers conducting economic analyses have two sources of VA cost information: DSS and HERC. Each data set has limitations. The HERC costs only vary by LOS. Although LOS is a powerful predictor of cost, the HERC data are inappropriate to use in economic analyses, where the intervention might affect costs but not LOS. The DSS data are borne from an activity-based cost methodology. We expect that many people will use the DSS cost data because this method is the gold standard. For researchers who use DSS costs, particular attention needs to be placed on identifying outliers. For FY01, we found a maximum cost per day of $\$ 22,633$; this outlier may be a data error and it merits further investigation. To identify outliers, researchers can divide the DSS discharge costs by LOS to estimate an average daily cost. Because LOS explains $>80$ percent of the variance, dividing costs by LOS will remove most of the variation. Researchers can then examine the distribution of average daily costs to identify low- or high-cost outliers. Researchers can also use the DSS or HERC costs in the primary analysis and then use the other data set in a sensitivity analysis.

The UDSMR data suggest that the daily cost of inpatient rehabilitation from non-VA providers was approximately $\$ 763$ in 2001 dollars. This may be sufficiently precise for some researchers to use in cost-effectiveness analyses. Researchers needing more precise estimates can use the regression coefficients in Table 2 to estimate costs. Interested readers can see Wagner et al. for an 
example and discussion of the limitations of this approach [32]. The daily cost of inpatient rehabilitation may be changing with the enactment of Medicare's prospective payment system. Future research will be needed to see if and how prospective payment has affected inpatient rehabilitation costs. In theory, the financial incentives behind prospective payment will push providers to minimize LOS and encourage greater use of outpatient services.

\section{LIMITATIONS}

Our analyses have several limitations. We cannot verify the accuracy of total charges in the UDSMR data. The large sample of hospitals from which the data are derived, however, minimizes bias resulting from coding errors in any given facility. A second limitation was incomplete matching across VA data sources. Approximately 24 percent of VA records could not be matched to FIM scores. Although this raises the possibility of selection bias, we do not see an obvious connection between FIM scores and the direction of the potential bias.

\section{CONCLUSIONS}

Our analyses of HERC and DSS suggest that neither data set is ideal for all research on VA rehabilitation care. Researchers conducting cost-effectiveness analyses where rehabilitation is not a primary end point and only a small percentage of patients use rehabilitation will probably find an average daily cost sufficiently precise. However, other researchers working in other clinical areas, such as stroke or fall prevention, may need more precise cost estimates. In these circumstances, VA researchers could use DSS data with caution.

More research is needed on the variation in the DSS cost per day. Non-VA researchers may need to use microcost methods to estimate costs, although these researchers will need to pay attention to Medicare's recent introduction of prospective payment because this may affect who is admitted to the rehabilitation hospital and the cost of care they receive.

\section{ACKNOWLEDGMENTS}

We would like to thank Dick Linn and Carl Granger for advice and comments.
This material was based on work supported by the VA Rehabilitation Research and Development Service, Rehabilitation Outcomes Research Center grant O3047C.

The authors have declared that no competing interests exist.

\section{REFERENCES}

1. Wagner TH, Chen S, Yu W, Barnett PG. HERC's average cost data for VA inpatient Care: 1998-2004. Menlo Park (CA): Department of Veterans Affairs Health Economics Resource Center; 2005.

2. Ashton CM, Souchek J, Petersen NJ, Menke TJ, Collins TC, Kizer KW, Wright SM, Wray NP. Hospital use and survival among Veterans Affairs beneficiaries. N Engl J Med. 2003;349(17):1637-46. [PMID: 14573736]

3. Chen S, Smith MW, Wagner TH, Barnett PG. Spending for specialized mental health treatment in The VA: 1995-2001. Health Aff (Millwood). 2003;22(6):256-63.

[PMID: 14649454]

4. Chen S, Wagner TH, Barnett PG. The effect of reforms on spending for veterans' substance abuse treatment, 19931999. Health Aff (Millwood). 2001;20(4):169-75. [PMID: 11463073]

5. Yu W, Ravelo A, Wagner TH, Barnett PG. The relationships among age, chronic conditions, and healthcare costs. Am J Manag Care. 2004;10(12):909-16. [PMID: 15617366]

6. Barnett PG, Rodgers JH. Use of the Decision Support System for VA cost-effectiveness research. Med Care. 1999;37(4 Suppl VA):AS63-70. [PMID: 10217386]

7. Wagner TH, Velez J. A Comparison for inpatient costs from the HERC and DSS National Data Extract Sata Sets. Menlo Park (CA): Department of Veterans Affairs Health Economics Resource Center; 2004.

8. Luke RD. Dimensions in hospital case mix measurement. Inquiry. 1979;16(1):38-49. [PMID: 155039]

9. Harada N, Kominski G, Sofaer S. Development of a resource-based patient classification scheme for rehabilitation. Inquiry. 1993;30(1):54-63. [PMID: 8454315]

10. Burnett DM, Cifu DX, Kolakowsky-Hayner S, Kreutzer JS. Predicting "charge outliers" after spinal cord injury: A multicenter analysis of demographics, injury characteristics, outcomes, and rehabilitation charges. Arch Phys Med Rehabil. 2001;82(1):114-19. [PMID: 11239296]

11. Brosseau L, Philippe P, Potvin L, Boulanger YL. Poststroke inpatient rehabilitation. I. Predicting length of stay. Am J Phys Med Rehabil. 1996;75(6):422-30. [PMID: 8985105]

12. Stineman MG, Hamilton BB, Goin JE, Granger CV, Fiedler RC. Functional gain and length of stay for major rehabilitation impairment categories. Patterns revealed by function 
related groups. Am J Phys Med Rehabil. 1996;75(1):68-78. [PMID: 8645443]

13. Heinemann AW, Linacre JM, Wright BD, Hamilton BB, Granger C. Prediction of rehabilitation outcomes with disability measures. Arch Phys Med Rehabil. 1994;75(2): 133-43. [PMID: 8311668]

14. Rondinelli RD, Murphy JR, Wilson DH, Miller CC. Predictors of functional outcome and resource utilization in inpatient rehabilitation. Arch Phys Med Rehabil. 1991;72(7): 447-53. [PMID: 1905529]

15. Roth EJ, Lovell L, Harvey RL, Bode RK, Heinemann AW. Stroke rehabilitation: Indwelling urinary catheters, enteral feeding tubes, and tracheostomies are associated with resource use and functional outcomes. Stroke. 2002;33(7): 1845-50. [PMID: 12105364]

16. Reker DM, O’Donnell JC, Hamilton BB. Stroke rehabilitation outcome variation in Veterans Affairs rehabilitation units: Accounting for case-mix. Arch Phys Med Rehabil. 1998;79(7):751-57. [PMID: 9685086]

17. Stineman MG, Ross RN, Hamilton BB, Maislin G, Bates B, Granger CV, Asch DA. Inpatient rehabilitation after stroke: A comparison of lengths of stay and outcomes in the Veterans Affairs and non-Veterans Affairs health care system. Med Care. 2001;39(2):123-37. [PMID: 11176550]

18. Stineman MG, Escarce JJ, Goin JE, Hamilton BB, Granger CV, Williams SV. A case-mix classification system for medical rehabilitation. Med Care. 1994;32(4):366-79. [PMID: 8139301]

19. Carter GM, Relles DA, Ridgeway GK, Rimes CM. Measuring function for Medicare inpatient rehabilitation payment. Health Care Financ Rev. 2003;24(3):25-44. [PMID: 12894633]

20. Carter GM, Buchanan JL, Buntin MB, Hayden O, Paddock S, Relles DA, Ridgeway G, Totten M, Wynn B. Executive summary for the analyses for the initial implementation of the inpatient rehabilitation facility prospective payment system. Santa Monica (CA): RAND; 2002.

21. Harvey RL, Roth EJ, Heinemann AW, Lovell LL, McGuire JR, Diaz S. Stroke rehabilitation: Clinical predictors of resource utilization. Arch Phys Med Rehabil. 1998;79(11): 1349-55. [PMID: 9821892]

22. Cowen TD, Meythaler JM, DeVivo MJ, Ivie CS 3rd, Lebow J, Novack TA. Influence of early variables in trau- matic brain injury on functional independence measure scores and rehabilitation length of stay and charges. Arch Phys Med Rehabil. 1995;76(9):797-803. [PMID: 7668948]

23. Andrews AW, Bohannon RW. Discharge function and length of stay for patients with stroke are predicted by lower extremity muscle force on admission to rehabilitation. Neurorehabil Neural Repair. 2001;15(2):93-97.

[PMID: 11811257]

24. Cifu DX, Huang ME, Kolakowsky-Hayner SA, Seel RT. Age, outcome, and rehabilitation costs after paraplegia caused by traumatic injury of the thoracic spinal cord, conus medullaris, and cauda equina. J Neurotrauma. 1999; 16(9):805-15. [PMID: 10521140]

25. Gold MR, Siegel JE, Russell LB, Weinstein MC. Costeffectiveness in health and medicine. Oxford (England): Oxford University Press; 1996.

26. Yu W, Wagner TH, Chen S, Barnett PG. Average cost of VA rehabilitation, mental health, and long-term hospital stays. Med Care Res Rev. 2003;60(3 Suppl):40S-53S. [PMID: 15095545]

27. Duan N. Smearing estimate: A nonparametric retransformation method. J Am Stat Assoc. 1983;78(383):605-10.

28. Ai C, Norton EC. Standard errors for the retransformation problem with heteroscedasticity. J Health Econ. 2000; 19(5):697-718. [PMID: 11184800$]$

29. Blough DK, Madden CW, Hornbrook MC. Modeling risk using generalized linear models. J Health Econ. 1999; 18(2):153-71. [PMID: 10346351]

30. Gertler PJ, Waldman DM. Quality-adjusted cost functions and policy evaluation in the nursing home industry. J Polit Econ. 1992;100(6):1232-56.

31. Frank RG, Berndt ER, Busch SH. Price indexes for the treatment of depression. Washington (DC): Brookings Institution Press; 1999.

32. Wagner TH, Chen S, Barnett PG. Using average cost methods to estimate encounter-level costs for medical-surgical stays in the VA. Med Care Res Rev. 2003;60(3 Suppl): 15S-36S. [PMID: 15095543]

Submitted for publication October 19, 2005. Accepted in revised form September 20, 2006. 